arrangement of polarizers and half-waveplate retarders. The technique can be used as a non-invasive scanning probe for detecting small details in the cell.

"Because of the simplicity and benefits, any confocal fluorescence light microscope should have a STED option. I am convinced that this will be the case in the foreseeable future," stated Hell at the end of his talk. He also added that brighter, faster and more compact STED systems are anticipated.

Continuing on the same subject, Egidijus Auksorius from Imperial College London presented the Imperial team's results on a commercial confocal scanning microscope equipped with STED, which is able to perform fluorescence lifetime imaging and fluorescence resonance energy transfer studies ${ }^{6}$. The team has acquired images with a resolution beyond the far-field diffraction limit in both the lateral and axial directions.

In near-field optics, tip-enhanced Raman scattering (TERS) microscopy is now developing at a rapid pace. This near-field optical microscopy scheme relies on Raman scattering enhanced by the plasmonic effects at the apex of a metallic tip to probe fields at resolutions beyond the diffraction limit of light. It is already receiving attention from the semiconductor industry for nanoimaging of strained silicon devices.
"The best resolution achieved by tip-enhanced coherent anti-Stokes Raman scattering remains around $10-15 \mathrm{~nm}$ using a laser at $800 \mathrm{~nm}$ [see ref. 7]," said Kawata, who gave a talk on imaging by exploiting plasmonics and going beyond the spatial limits it sets. However, he pointed out that by incorporating atomic force microscopy into TERS, mechanical interactions at molecular distances can create localized structural deformation on the sample perturbing the Raman modes at a much higher spatial resolution ${ }^{8}$. This potentially provides a means to drastically improve the spatial resolution of TERS. He also commented on the idea of using chemical interactions between the tip molecules and sample molecules to provide higher spatial resolution for single-molecule imaging 9 .

"Applications of TERS in nanoimaging of cells, proteins on membranes, drug distribution, mitochondria and organelles, as well as in material studies, and optimization of design and fabrication technology, are anticipated," commented Kawata. "However, a breakthrough concept is still needed to beat the plasmonic limit for higher resolution, and thereafter spectral range, signal [strength] and reproducibility stand as other challenges to be overcome."

Although new barriers are arriving to replace the old ones, a common view expressed at the meeting is that high-resolution optical imaging technologies have a very bright future. They are already allowing scientists to probe samples with unprecedented resolution and an optical approach often has advantages over alternative technologies, which can be very expensive or bulky.

Kawata commented that optical microscopy is preferred, as it offers colour and sectional imaging that can not be obtained from electron, atomic force or scanning tunnelling microscopes.

As Hell neatly summarized, "Light is gentle. For life sciences applications, optics will remain the best choice over other microscopic technologies owing to its non-invasiveness, unique selectivity, specificity and sensitivity for visualizing the biomolecules of interest."

The next Focus on Microscopy event will be held in Krakow, Poland, from 5-8 April 2009 (ref. 1).

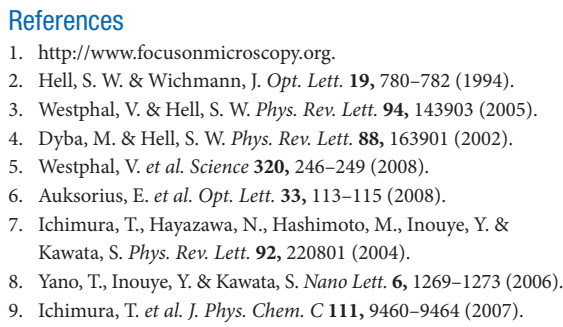

\title{
HIGH-HARMONIC GENERATION
}

\section{Observing quantum interference}

High-harmonic generation (HHG) is a useful technique for producing extreme UV (EUV) light or trains of attosecond pulses and is now a popular area of research. The process, which results from the interaction of an intense laser field with a collection of atoms or molecules, is often described by classical physics, but more recently models based on quantum mechanics have been developed. The strength of the process is characterized by a harmonic dipole moment, which can be described as a sum of different quantum path contributions, which represent electron trajectories. The relative phase between the different quantum paths (electron trajectories) leads to interference in the total single-atom dipole moment.

A collaboration of scientists from Switzerland, the UK and France now claim to have observed this quantum path interference (QPI) for the first time, by studying the intensity direct observation.

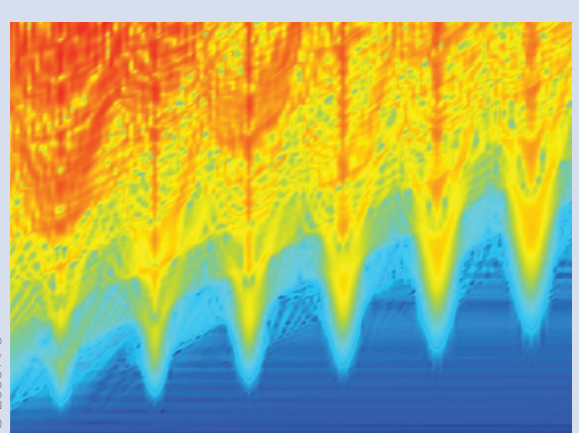

University and the CNRS-CEA University of Bordeaux and CEA Saclay in France say that their measurements have sufficient contrast to resolve QPI in the generated harmonics (see image). The measurements also provide access to the relative quantum path phases that are needed to reconstruct the full atom dipole moment.

By slightly changing the intensity of the incident laser beam the team were able to control the quantum paths on an attosecond timescale. For dependence of HHG in an argon gas jet (Phys. Rev. Lett. 100, 143902; 2008).

Such QPI is hard to observe because temporal and spatial averaging usually blurs the effects on a macroscopic scale. However, the researchers used spectral and far-field spatial filtering to overcome this problem and allow

The team from ETH Zurich, the Clarendon Laboratory at Oxford example a $10 \%$ change in incident laser intensity was able to shift the QPI from constructive to destructive for the 15 th harmonic. insight into the ultrafast electron dynamics involved in the emission process and is the first step towards a direct experimental characterization of a single atom dipole.

Oliver Graydon
The research provides a valuable 